\title{
Giant Modal Gain Coefficients in Colloidal II-VI Nanoplatelets
}

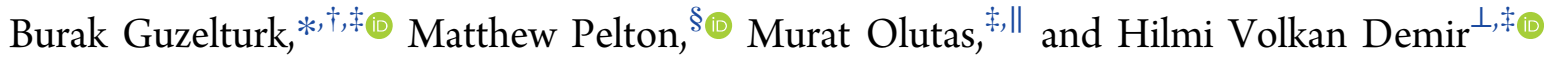 \\ ${ }^{\dagger}$ Stanford Institute for Materials and Energy Sciences, SLAC National Accelerator Laboratory, Menlo Park, California 94025, United \\ States \\ ${ }^{\ddagger}$ Department of Electrical and Electronics Engineering, Department of Physics, UNAM - Institute of Materials Science and \\ Nanotechnology, Bilkent University, Ankara 06800 Turkey \\ ${ }^{\S}$ Department of Physics, University of Maryland, Baltimore County, Baltimore, Maryland 21250, United States \\ "Department of Physics, Bolu Abant Izzet Baysal University, Bolu 14030, Turkey \\ ${ }^{\perp}$ Luminous! Center of Excellence for Semiconductor Lighting and Displays, School of Electrical and Electronic Engineering, School \\ of Physical and Mathematical Sciences, School of Materials Science and Engineering, Nanyang Technological University, Nanyang \\ Avenue, Singapore 639798, Singapore
}

Supporting Information

ABSTRACT: Modal gain coefficient is a key figure of merit for a laser material. Previously, net modal gain coefficients larger than a few thousand $\mathrm{cm}^{-1}$ were achieved in II-VI and III-V semiconductor gain media, but this required operation at cryogenic temperatures. In this work, using pump-fluencedependent variable-stripe-length measurements, we show that colloidal $\mathrm{CdSe}$ nanoplatelets enable giant modal gain coefficients at room temperature up to $6600 \mathrm{~cm}^{-1}$ under pulsed optical excitation. Furthermore, we show that exceptional gain performance is common to the family of CdSe nanoplatelets, as shown by examining samples having different vertical thicknesses and lateral areas. Overall, colloidal II-VI nanoplatelets with superior optical gain properties are

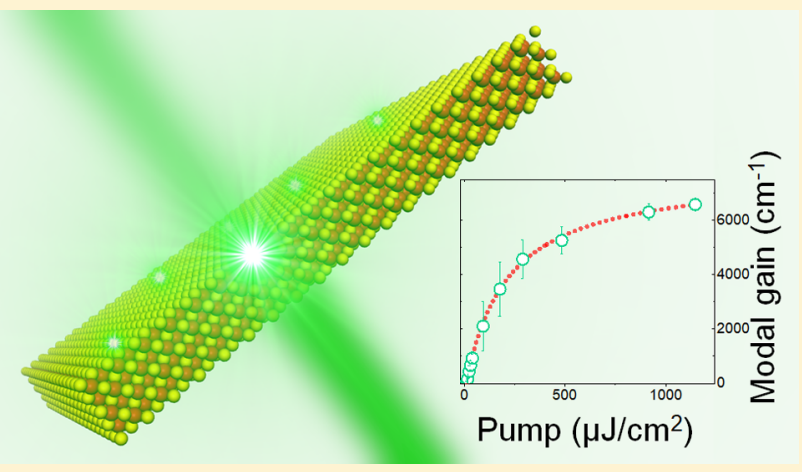
promising for a broad range of applications, including high-speed light amplification and loss compensation in plasmonic photonic circuits.

KEYWORDS: Colloidal nanoplatelets, colloidal quantum wells, CdSe, modal gain coefficient, variable stripe length method, optical gain

$\mathrm{O}$ ver the past decade, colloidal CdSe nanoplatelets (NPLs), also alternatively known as colloidal quantum wells, joined the family of II-VI semiconductor nanocrystals as a promising new member ${ }^{1}$ and have attracted great interest for optoelectronic applications owing to their superior optical properties, including spectrally narrow photoluminescence, ${ }^{2}$ strong light absorption, ${ }^{3}$ and polarized light emission. ${ }^{4}$ Recent reports ${ }^{5-12}$ identified a key application for NPLs as gain media for optically pumped lasers. To date, NPL heterostructures exhibited remarkable optical gain performance, including low gain thresholds ${ }^{5}\left(\sim 8 \mu \mathrm{J} / \mathrm{cm}^{2}\right)$ and long gain lifetimes ${ }^{13}(\sim 400$ ps). Moreover, initial reports showed appreciably large modal gain coefficient $\left(\sim 650 \mathrm{~cm}^{-1}\right)^{5,6}$ in the NPLs, significantly larger than those found for conventional quantum dots. ${ }^{14,15}$

Modal gain coefficient is an important benchmarking parameter for a gain medium and quantifies how much light is amplified per unit length. CdSe NPLs possess significantly large linear ${ }^{3,16}$ and nonlinear ${ }^{17,18}$ absorption cross-sections due to their giant oscillator strength. ${ }^{19}$ This suggests the possibility of a large stimulated emission cross-section and associated large modal gain coefficients. However, the limits of optical gain using CdSe NPLs have not been elucidated to date. Here we show that CdSe NPLs attain giant net modal gain coefficients as high as $\sim 6600 \mathrm{~cm}^{-1}$ at room temperature. This net modal gain exceeds those reported for any other gain media at room temperature so far. This makes these solution processable NPLs highly attractive for applications that require high modal gain, such as lossy plasmonic photonic structures ${ }^{20}$ and parity-time-symmetric photonic designs. ${ }^{21}$

In this work, we synthesized CdSe NPLs with different vertical thicknesses and lateral areas based on previous recipes. ${ }^{2,17,22}$ In Figure 1, we show optical absorption and photoluminescence (PL) spectra of CdSe NPLs that have 4 monolayer (ML) vertical thickness. The two absorption peaks at 511 and $480 \mathrm{~nm}$ are due to excitonic absorption associated with electron-heavy hole and electron-light hole transitions, respectively. ${ }^{2}$ The absorption profile shows a steplike density

Received: September 26, 2018

Revised: December 11, 2018

Published: December 12, 2018 
(a)

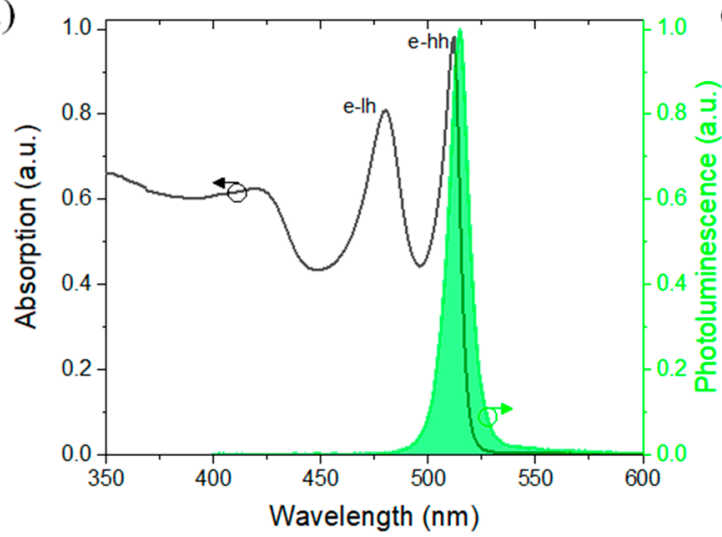

(b)

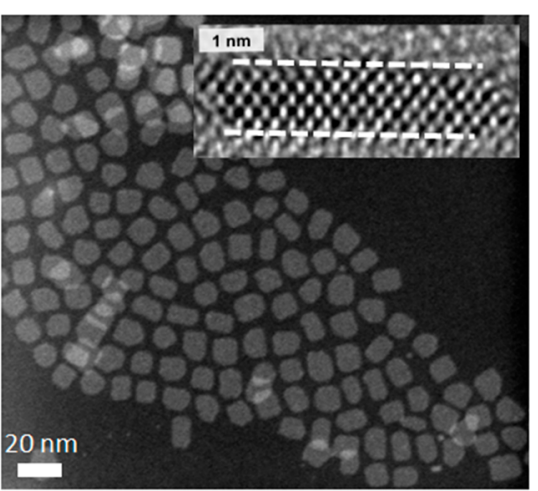

Figure 1. (a) Absorbance and photoluminescence spectra (normalized) and (b) HAADF-STEM image of CdSe nanoplatelets (NPLs) having 4 ML vertical thickness. The inset in (b) shows a high-resolution TEM cross-section image of a 4 ML thick NPL.

of states due to one-dimensional quantum confinement in these materials. ${ }^{17}$ The PL peak is at $513 \mathrm{~nm}$ (when the NPLs are dissolved in toluene) and the emission profile is quite narrow (with a full width at half-maximum of $\sim 8 \mathrm{~nm}$ at room temperature) due to atomically flat surfaces and atomic-level monodispersity in thickness. ${ }^{23}$ Figure $1 \mathrm{~b}$ shows a high-angle annular dark-field scanning transmission electron microscopy (HAADF-STEM) image of the 4 monolayer thick CdSe NPLs. For this batch of sample, we find the average lateral size to be 9 $\mathrm{nm}$ by $12 \mathrm{~nm}$ and the vertical thickness to be $\sim 1.2 \mathrm{~nm}$ (see inset of Figure $1 \mathrm{~b}$ ).

We make solid thin films by spin-coating the NPLs on precleaned $1 \mathrm{~cm}$ by $1 \mathrm{~cm}$ quartz substrates to study amplified spontaneous emission (ASE). The postsynthesis cleaning procedure has been optimized, which allowed us to prepare NPL solid films that are close-packed and free of excess ligands. The film thickness of the samples studied here is $\sim 200$ $\mathrm{nm}$. When the NPLs form a close-packed solid film, their PL peak redshifts to $518-522 \mathrm{~nm}$ and the emission line width increases to $15 \mathrm{~nm}$. This redshift and broadening could arise from an increase in the effective dielectric function surrounding the NPLs, which would increase the dielectric screening and lead to inhomogeneous broadening. In addition, densely packed NPL thin-films could favor the formation of excimer states, ${ }^{24}$ which exhibit a red-shifted emission peak as compared to that of the free exciton emission. We excite the samples in a stripe geometry by focusing the pump beam via a cylindrical lens. We used a pulsed Ti:sapphire femtosecond laser (SpectraPhysics, Spitfire Pro) to excite the samples. The output of the laser is frequency doubled using a BBO crystal. The excitation photon energy is $3.1 \mathrm{eV}$, the pulse width is $\sim 150 \mathrm{fs}$, and the repetition rate is $1 \mathrm{kHz}$. Emission from the NPLs is detected by a fiber-coupled spectrometer and the fiber is positioned close the edge of the sample. Figure $2 \mathrm{a}$ shows the evolution of the emission spectrum as a function of the pump fluence. As the pump fluence is increased, a new peak rises at $532 \mathrm{~nm}$, which is due to ASE and indicates the presence of net optical gain in the material. The ASE peak is red-shifted as compared to the PL peak, which is consistent with previous reports suggesting biexcitons as the gain active species in the NPLs. ${ }^{7,25}$ Figure $2 \mathrm{~b}$ depicts the integrated emission intensity versus pump fluence marking the ASE threshold, which happens when the modal gain becomes equal to the loss of the system. In this sample, we find the ASE threshold to be 13 $\mu \mathrm{J} / \mathrm{cm}^{2}$, which is on par with the best previous report in CdSe

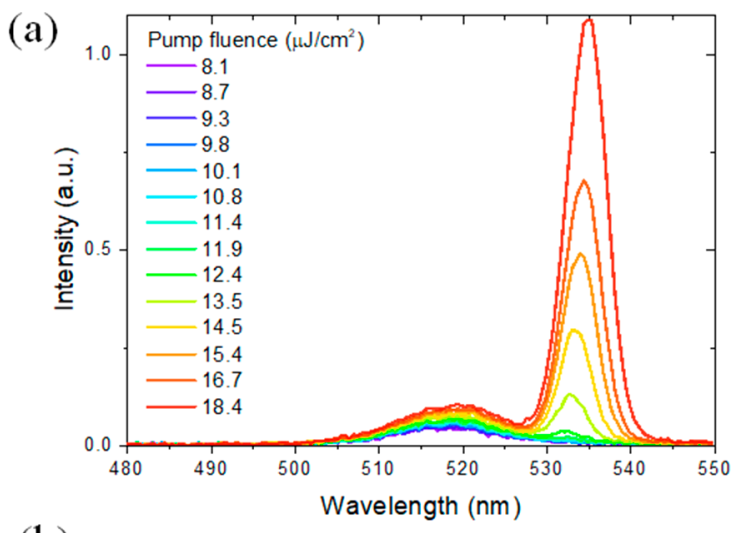

(b)

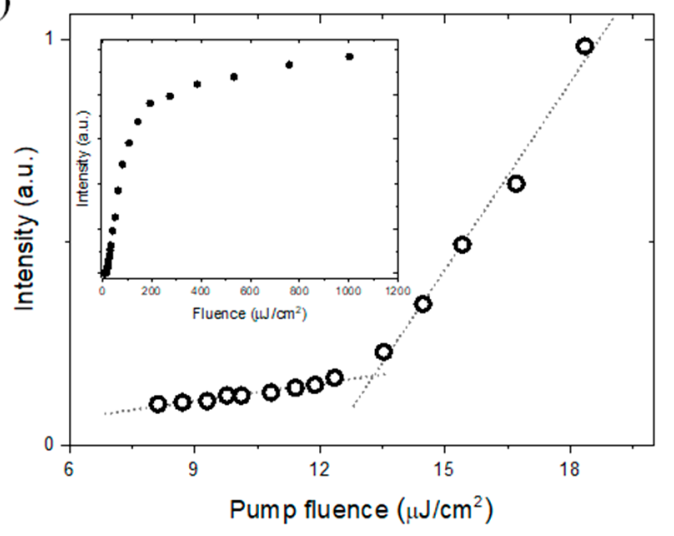

Figure 2. (a) Emission spectra of a thin film of CdSe nanoplatelets (NPLs) under increasing pump fluence. An amplified spontaneous emission (ASE) peak emerges at $532 \mathrm{~nm}$ when the fluence is above 13 $\mu \mathrm{J} / \mathrm{cm}^{2}$. (b) Integrated emission intensity versus pump fluence showing a threshold behavior and transition from spontaneous photoluminescence to ASE. Inset shows the integrated emission intensity over a larger excitation fluence range.

$\mathrm{NPLs}^{7}$ and is within a factor of 3 as compared to the best reports in colloidal nanocrystals (e.g., cesium lead halide perovskite nanocrystals ${ }^{26}$ and giant-shell $\mathrm{CdSe} / \mathrm{CdS}$ quantum dots $^{27}$ ) to date.

Now we turn our focus to net modal gain coefficients measured by the variable stripe length (VSL) method, ${ }^{28,29}$ which has been commonly employed to characterize modal gain in a broad range of solid-state gain media such as quantum dots, $^{15}$ organic semiconductors, ${ }^{30}$ and single-crystal bulk 
(a)

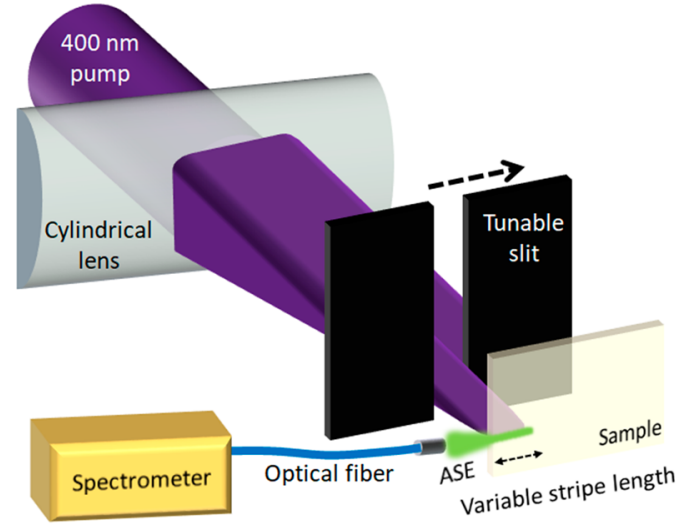

(c)

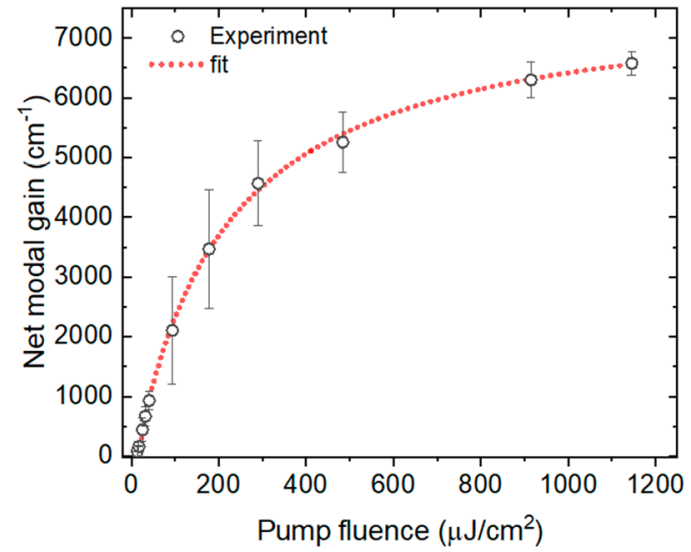

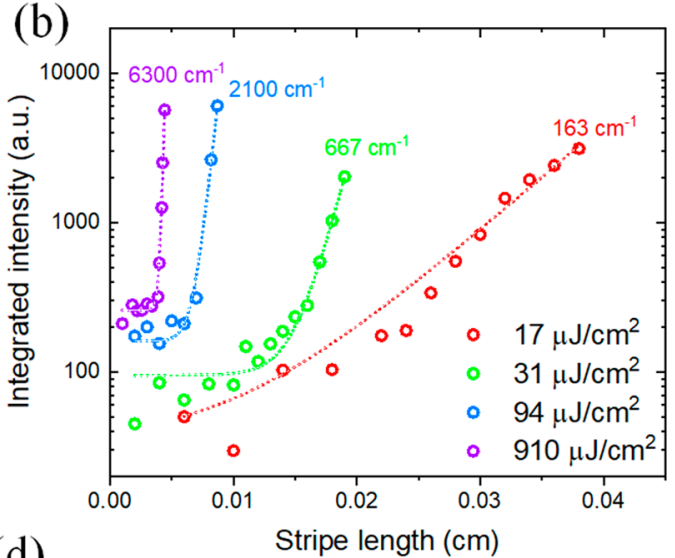

(d)

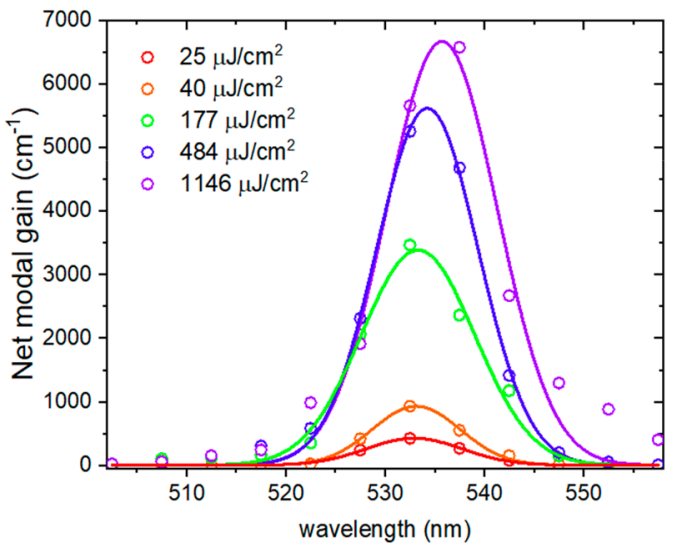

Figure 3. (a) Schematic representation of the experimental setup for the VSL measurements. (b) Integrated emission intensity (log-scale) from the sample as a function of the stripe length for five different pump intensities. Fits are performed using eq 1. (c) Net modal gain coefficient as a function of the pump fluence. A saturation curve fits the experimental data with a saturation fluence of $195 \mu \mathrm{J} / \mathrm{cm}^{2}$. The maximum net modal gain is measured to be $\sim 6600 \mathrm{~cm}^{-1}$. (d) Modal gain spectra measured at five different pump intensities and fitted by Gaussian functions.

materials. ${ }^{31}$ As shown in Figure 3a, the sample is excited in a stripe geometry, and we change the stripe length via a tunable slit. To minimize diffraction effects, ${ }^{32}$ the slit is positioned close to the sample $(\sim 5 \mathrm{~mm})$. To ensure that the stripe begins from the edge of the sample, one side of the slit is fixed while the other side is moved. Figure $3 \mathrm{~b}$ shows the integrated emission intensity (in log-scale) as a function of the stripe length measured under four different pump intensities. Net gain in the sample provides amplification along the stripe direction, hence the integrated emission intensity increases exponentially as a function of the stripe length. This relationship is commonly expressed as ${ }^{28}$

$$
\begin{aligned}
& I_{\text {emission }}(l)=\frac{A}{g_{\text {net }}}\left(e^{g_{\text {net }} l}-1\right) \\
& g_{\text {net }}=g_{\text {modal }}-\alpha
\end{aligned}
$$

where $l$ is the stripe length, $A$ is the spontaneous emission growth parameter, $g_{\text {net }}$ is the net modal gain coefficient, $g_{\text {modal }}$ is the modal gain coefficient, and $\alpha$ is the waveguide loss coefficient. Fits using eq 1 match well with the experimental data (see Figure $3 \mathrm{~b}$ ). For example, we find $g_{\text {net }}$ to be $163 \pm 10$ $\mathrm{cm}^{-1}$ at a fluence of $17 \mu \mathrm{J} / \mathrm{cm}^{2}$. As the pump fluence is increased, the integrated emission intensity starts to rise at a faster rate. This implies that $g_{\text {net }}$ rises as the pump intensity is increased. For example, we find $g_{\text {net }}$ to be $6300 \pm 300 \mathrm{~cm}^{-1}$ at $910 \mu \mathrm{J} / \mathrm{cm}^{2}$.
Figure $3 \mathrm{c}$ shows the evolution of net modal gain, $g_{\text {net }}$ as a function of the pump fluence. Here, the average of four different measurements on the same sample for each pump intensity is considered to provide statistics, as highlighted via error bars in Figure 3c. At low pump fluences $\left(15-60 \mu \mathrm{J} / \mathrm{cm}^{2}\right)$, $g_{\text {net }}$ increases linearly as a function of the fluence, whereas above this level $g_{\text {net }}$ starts to deviate from the linear relationship and saturates which is consistent with the integrated emission intensity from the sample shown in the inset of Figure $2 \mathrm{~b}$. This behavior can be fitted by a saturation curve (see Figure 3c) with a saturation fluence of $195 \mu \mathrm{J} / \mathrm{cm}^{2}$. The maximum $g_{\text {net }}$ is as high as $6600 \pm 350 \mathrm{~cm}^{-1}$.

To validate these giant modal gain coefficients, we reconsider the constraints of the VSL method. Equation 1 is valid when the modal gain of the material is independent of the stripe length. However, in the case of very large gain, ASE itself can deplete the population inversion within the excitation stripe, hence integrated emission intensity may saturate as the stripe length is increased. Previous reports ${ }^{33,34}$ showed that saturation length $\left(L_{\text {sat }}\right)$ times net modal gain $\left(L_{\text {sat }} \times g_{\text {net }}\right)$ is on the order of $\sim 10$. Thus, when $g_{\text {net }}$ is $5000 \mathrm{~cm}^{-1}$, one could expect $L_{\text {sat }}$ to be on the order of $\sim 20 \mu \mathrm{m}$. Because of this, eq 1 would be valid only for a short-range of stripe lengths before the emitted intensity saturates as a function of the stripe length. To understand this point better, we also considered a full analytical form derived by Cerdán et al., ${ }^{35}$ which takes into account the stripe length dependent saturation (see SI). We 
fitted the same experimental data for the highest pump intensity case using both the full analytical form (eq S2) and eq 1 (see Figure S1). The full analytical form gives $g_{\text {net }}=6530$ $\mathrm{cm}^{-1}$, which is in very good agreement with the value of $\mathrm{g}_{\text {net }}$ fitted by eq 1 . When using eq 1 , we limited the stripe length span to $\sim 40 \mu \mathrm{m}$ to stay away from the saturation region.

We also frequency-resolve the net modal gain coefficients (see Figure 3d) for five different pump fluences. Here, $g_{\text {net }}(\lambda)$ follows the ASE profile, as expected. The $g_{\text {net }}$ spectrum slightly redshifts (from 532 to $536 \mathrm{~nm}$ ) and broadens (from 11 to 13 $\mathrm{nm}$ ) as the pump fluence is increased. This redshift at increased excitation fluence is likely to originate from manybody Coulomb interactions causing band gap renormalization and electron-hole plasma screening, as previously observed in epitaxial quantum-well-based gain media. ${ }^{36}$ The narrow bandwidth of the gain even at very high pump intensity is an important feature enabling giant modal gain coefficients in these NPLs, because a large density of population inversion (i.e., multiple biexcitons per NPL) can be generated and sustained in a highly concentrated spectral region.

We also investigate the net modal gain coefficients in CdSe NPLs having different vertical thickness and lateral area. Figure 4a shows the ASE spectra of NPLs having 3, 4, and $5 \mathrm{ML}$
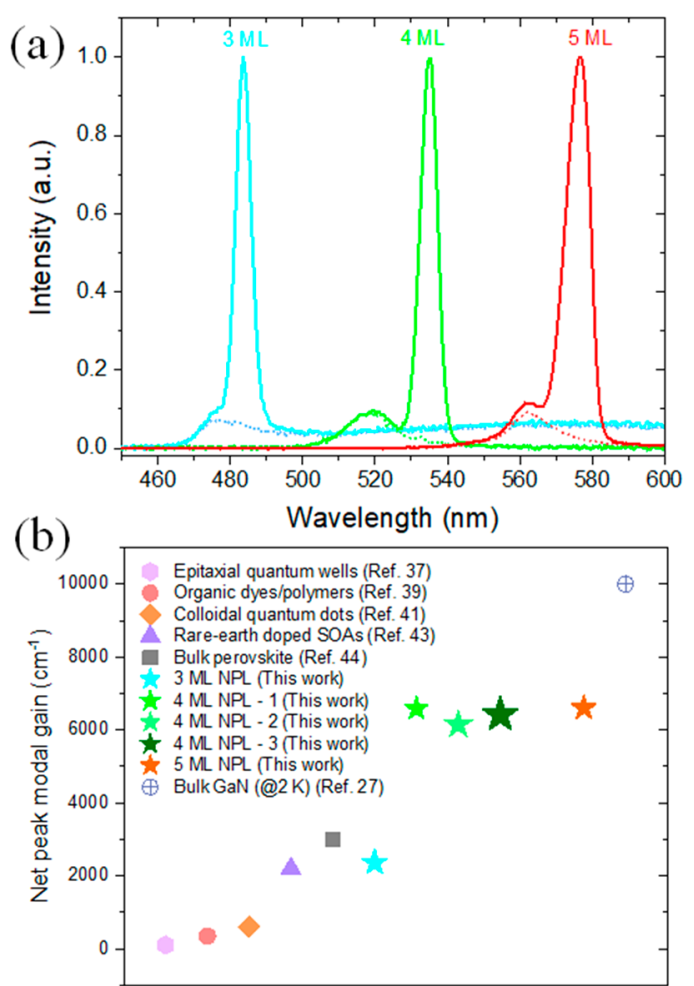

Figure 4. (a) Amplified spontaneous emission (ASE) spectra of nanoplatelets (NPLs) with 3, 4, and 5 ML vertical thickness. (b) Peak net modal gain coefficients in the NPLs with different vertical thicknesses and lateral areas. Peak net modal gains reported in other systems are also compared here. The empty symbol indicates a measurement at cryogenic temperatures.

thickness with ASE peaks at 480, 532, and $575 \mathrm{~nm}$, respectively. The optical gain covers an important range falling within the "green gap" region, which has been difficult to access with conventional epitaxial semiconductor materials. Via heterostructuring and alloying of the NPLs, the spectral gain coverage could be extended even further. ${ }^{37,38}$ In addition, we study $4 \mathrm{ML}$ thick NPLs with different lateral extent (4 ML NPL-1: $9 \mathrm{~nm}$ by $11 \mathrm{~nm}$; ML NPL-2: $11 \mathrm{~nm}$ by $14 \mathrm{~nm}$; and 4 ML NPL-3: $14 \mathrm{~nm}$ by $18 \mathrm{~nm}$ ), to check the dependence of the maximum achievable net modal gains on the lateral area. We perform VSL measurements on these different NPL samples (see SI for the VSL curves measured in the high-fluence range and respective fits using eq 1). Among different vertical thickness NPLs, the 3 ML thick one exhibits the smallest $g_{\text {net }}$ of about $2400 \mathrm{~cm}^{-1}$. Smaller modal gain in the $3 \mathrm{ML}$ sample is mainly associated with its poor stability, where the ASE peak intensity immediately starts decreasing when the pump fluence is above the ASE threshold. On the other hand, encapsulated 4 and 5 ML NPL samples exhibited significantly improved stability. Under an excitation fluence of $30 \mu \mathrm{J} / \mathrm{cm}^{2}$, the ASE peak remained stable for $60 \mathrm{~min}$ under continuous exposure to the pump, whereas the ASE peak was stable for 3 min under an excitation fluence of $1 \mathrm{~mJ} / \mathrm{cm}^{2}$. Among $4 \mathrm{ML}$ samples with different lateral areas, we also do not see any significant difference in the maximum $g_{\text {net }}$. Also, $5 \mathrm{ML}$ sample exhibited a maximum $g_{\text {net }}$ that is very similar to that of the $4 \mathrm{ML}$ samples. These indicate that stable NPLs (both 4 and 5 ML thick ones) offer giant modal gain independent of their vertical thickness and lateral area. Overall, the maximum measured $g_{\text {net }}$ is $\sim 6600$ $\mathrm{cm}^{-1}$.

Figure $4 \mathrm{~b}$ shows the maximum $g_{\text {net }}$ achieved in these NPL samples along with maximum $g_{\text {net }}$ measured in other material systems. Previously, II-VI (e.g., $\mathrm{CdS}$ and $\mathrm{ZnO})^{39}$ and III-V (e.g., $\mathrm{GaN}^{31}$ an InGaAsP $\mathrm{P}^{40,41}$ ) semiconductor materials in their bulk form exhibited very large modal gain up to $10,000 \mathrm{~cm}^{-1}, 31$ but these were all measured at low temperatures $(T<10 \mathrm{~K})$. Quantum confined epitaxial systems such as III-V quantum wells ${ }^{42}$ and $\operatorname{dots}^{43}$ were shown to exhibit modal gain in the range of 10 to $\sim 100 \mathrm{~cm}^{-1}$, although their intrinsic material gain can be very large $\left(>10,000 \mathrm{~cm}^{-1}\right)$. Organic gain media such as organic dyes ${ }^{44}$ and conjugated polymers ${ }^{45}$ also offered limited modal gain up to $350 \mathrm{~cm}^{-1}$. In colloidal quantum dots and nanorods, the maximum $g_{\text {net }}$ was reported ${ }^{15,46,47}$ to be $\sim 600 \mathrm{~cm}^{-1}$. Semiconductor optical amplifiers based on rare earth-doped glasses ${ }^{48}$ showed relatively large modal gains reaching a level of $2400 \mathrm{~cm}^{-1}(\sim 1000 \mathrm{~dB} / \mathrm{cm}$ for an amplifier length of $25 \mu \mathrm{m})$. More recently, polycrystalline methylammonium lead halide perovskite thin-films ${ }^{49}$ showed a net modal gain of $3000 \mathrm{~cm}^{-1}$. To the best of our knowledge, colloidal CdSe NPLs attain the largest net modal gain coefficient measured at room temperature among any other optical gain system reported so far. ${ }^{50}$

Spectrally narrow and giant modal gain was theoretically predicted for strongly quantum confined systems by Asada et al. ${ }^{51}$ but was not previously possible to achieve using colloidal quantum dots. To date, colloidal quantum dots suffered from small modal gain coefficients due to short-lived gain associated with nonradiative Auger recombination. ${ }^{52}$ In addition, the material gain was small in these quantum dots, because the QDs cannot support multiple excitons at their band edges. Because of this, modal gain coefficients for these materials saturate at pump intensities approximately twice the net gain threshold. ${ }^{27}$ On the other hand, NPLs can sustain large gain even at much higher pump levels ( $\sim 2$ orders of magnitude above the net gain threshold). This indicates that material gain $\left(g_{\text {mat }}\right)$ is also large: $g_{\text {net }}=\Gamma \times g_{\text {mat }}$, where $\Gamma$ is the mode confinement factor. In the thin films having high volume packing density, we estimate ${ }^{53} \Gamma$ to be 0.43 by considering the thickness of the film ( $200 \mathrm{~nm}$, see Figure S3) and its effective 
refractive index $\left(\sim 1.7^{54}\right)$. Thus, the material gain of the NPL thin-film at room temperature is $\sim 15,300 \mathrm{~cm}^{-1}$, which is comparable to the best gain systems of bulk $\mathrm{GaN}^{31}$ and $\mathrm{GaAs}^{43}$ with material gains estimated to be $\sim 20,000 \mathrm{~cm}^{-1}$ at cryogenic temperatures. It is also on par with the best epitaxial quantum dot systems (e.g., InAs/ $/ \mathrm{GaAs}^{55}$ ) with a material gain of 15,000 $\mathrm{cm}^{-1}$ at room temperature. ${ }^{50} \mathrm{~A}$ recent communication also agrees well with the existence of large material gain in $\mathrm{CdSe}$ NPLs. ${ }^{56}$

From the application point of view, excitation pulse duration is an important parameter. Although continuous-wave (CW) or quasi-CW pumping is desired for practical applications, short-lived gain $(<10 \mathrm{ps})$ has posed a significant limitation in conventional quantum dot-based gain systems. ${ }^{57}$ CdSe NPLs have shown significantly longer gain lifetimes, ranging from $150^{9}$ to $400 \mathrm{ps}^{13}$ hence quasi-CW pumping is expected to be within reach for the NPLs. Indeed, recent reports including refs 7 and 10 showed that CW lasing is possible using the NPL-based gain media. This indicates that large intrinsic gain can be sustained under CW pumping, and Auger recombination is less detrimental as compared to conventional colloidal quantum dots. Under $\mathrm{CW}$ operation, heat dissipation and material stability would become important factors toward a sustainable optical gain. ${ }^{58}$

Overall, colloidal NPLs can reach a high density of population inversion, which indicates that each NPL can sustain multiple gain-active excitonic species. This suggests that multiexcitonic optical gain is extremely stable and nonradiative annihilation of biexcitons is minimized. The saturation effect in Figure $3 c$ can be attributed to either a phase-space filling effect, where the maximum number of biexcitons that can simultaneously survive within a NPL is limited due to the Pauli exclusion principle as predicted by Miller et al., ${ }^{59}$ or to exciton dissociation at high densities due to a Mott transition. ${ }^{60}$

In conclusion, NPLs combine the advantageous aspects of both colloidal systems (i.e., large mode confinement factor) and epitaxial semiconductors (i.e., large intrinsic material gain) and provide giant modal gain coefficients at room temperature. These materials possess high band-edge density of states and large exciton binding energies, allowing for highly efficient excitonic gain. Unlike in conventional colloidal quantum dots, exciton-exciton interactions are relatively unimportant in NPLs, simply because the spatial exciton density required for gain is lower. Importantly, solution processability of the NPLs means that they can be integrated into arbitrary substrates, which allows in principle for low-cost device fabrication and ease of processing. In addition, superior optical gain performance of these NPLs makes them exciting prospects for CW pumped colloidal lasers as well as for electrical injection.

\section{ASSOCIATED CONTENT}

\section{S Supporting Information}

The Supporting Information is available free of charge on the ACS Publications website at DOI: 10.1021/acs.nanolett.8b03891.

Full analytical form of the fitting function for the VSL data, comparison of the VSL fits using full form versus classical form, and VSL data measured using the samples mentioned in the main text (PDF)

\section{AUTHOR INFORMATION}

\section{Corresponding Author}

*E-mail: guzelturk@gmail.com. Phone.: +1 6505612481. ORCID

Burak Guzelturk: 0000-0003-1977-6485

Matthew Pelton: 0000-0002-6370-8765

Hilmi Volkan Demir: 0000-0003-1793-112X

\section{Notes}

The authors declare no competing financial interest.

\section{ACKNOWLEDGMENTS}

We gratefully acknowledge financial support from the Singapore National Research Foundation under the programs of NRF-NRFI2016-08. H.V.D. also acknowledges support from ESF-EURYI and TUBA.

\section{REFERENCES}

(1) Ithurria, S.; Dubertret, B. J. Am. Chem. Soc. 2008, 130 (49), 16504-16505.

(2) Ithurria, S.; Tessier, M. D.; Mahler, B.; Lobo, R. P. S. M.; Dubertret, B.; Efros, A. L. Nat. Mater. 2011, 10 (12), 936-941.

(3) Yeltik, A.; Delikanli, S.; Olutas, M.; Kelestemur, Y.; Guzelturk, B.; Demir, H. V. J. Phys. Chem. C 2015, 119 (47), 26768-26775.

(4) Gao, Y.; Weidman, M. C.; Tisdale, W. A. Nano Lett. 2017, 17 (6), 3837-3843.

(5) She, C.; Fedin, I.; Dolzhnikov, D. S.; Demortière, A.; Schaller, R. D.; Pelton, M.; Talapin, D. V. Nano Lett. 2014, 14 (5), 2772-2777.

(6) Guzelturk, B.; Kelestemur, Y.; Olutas, M.; Delikanli, S.; Demir, H. V. ACS Nano 2014, 8 (7), 6599-6605.

(7) Grim, J. Q.; Christodoulou, S.; Di Stasio, F.; Krahne, R.; Cingolani, R.; Manna, L.; Moreels, I. Nat. Nanotechnol. 2014, 9 (11), 891-895.

(8) Kelestemur, Y.; Guzelturk, B.; Erdem, O.; Olutas, M.; Gungor, K.; Demir, H. V. H. V. Adv. Funct. Mater. 2016, 26 (21), 3554.

(9) She, C.; Fedin, I.; Dolzhnikov, D. S.; Dahlberg, P. D.; Engel, G. S.; Schaller, R. D.; Talapin, D. V. ACS Nano 2015, 9 (10), 94759485.

(10) Yang, Z.; Pelton, M.; Fedin, I.; Talapin, D. V.; Waks, E. Nat. Commun. 2017, 8 (1), 143.

(11) Li, M.; Zhi, M.; Zhu, H.; Wu, W.-Y.; Xu, Q.-H.; Jhon, M. H.; Chan, Y. Nat. Commun. 2015, 6, 8513.

(12) Pelton, M. J. Phys. Chem. C 2018, 122 (20), 10659-10674.

(13) Guzelturk, B.; Kelestemur, Y.; Olutas, M.; Li, Q.; Lian, T.; Demir, H. V. J. Phys. Chem. Lett. 2017, 8 (21), 5317.

(14) Guzelturk, B.; Kelestemur, Y.; Gungor, K.; Yeltik, A.; Akgul, M. Z. M. Z.; Wang, Y.; Chen, R.; Dang, C.; Sun, H.; Demir, H. V. H. V. Adv. Mater. 2015, 27 (17), 2741-2746.

(15) Malko, A. V.; Mikhailovsky, A. A.; Petruska, M. A.; Hollingsworth, J. A.; Htoon, H.; Bawendi, M. G.; Klimov, V. I. Appl. Phys. Lett. 2002, 81 (7), 1303.

(16) Achtstein, A. W.; Antanovich, A.; Prudnikau, A.; Scott, R.; Woggon, U.; Artemyev, M. J. Phys. Chem. C 2015, 119, 2015620161.

(17) Olutas, M.; Guzelturk, B.; Kelestemur, Y.; Yeltik, A.; Delikanli, S.; Demir, H. V. H. V. ACS Nano 2015, 9 (5), 5041-5050.

(18) Scott, R.; Achtstein, A. W.; Prudnikau, A.; Antanovich, A.; Christodoulou, S.; Moreels, I.; Artemyev, M.; Woggon, U. Nano Lett. 2015, 15, 4985.

(19) Naeem, A.; Masia, F.; Christodoulou, S.; Moreels, I.; Borri, P.; Langbein, W. Phys. Rev. B: Condens. Matter Mater. Phys. 2015, 91 (12), 121302.

(20) Khurgin, J. B. Nat. Nanotechnol. 2015, 10 (1), 2-6.

(21) Peng, B.; Özdemir, Ş. K.; Lei, F.; Monifi, F.; Gianfreda, M.; Long, G. L.; Fan, S.; Nori, F.; Bender, C. M.; Yang, L. Nat. Phys. 2014, 10 (5), 394-398. 
(22) Guzelturk, B.; Olutas, M.; Delikanli, S.; Kelestemur, Y.; Erdem, O.; Demir, H. V. H. V. Nanoscale 2015, 7 (6), 2545-2551.

(23) Tessier, M. D.; Spinicelli, P.; Dupont, D.; Patriarche, G.; Ithurria, S.; Dubertret, B. Nano Lett. 2014, 14 (1), 207-213.

(24) Diroll, B. T.; Cho, W.; Coropceanu, I.; Harvey, S. M.; Brumberg, A.; Holtgrewe, N.; Crooker, S. A.; Wasielewski, M. R.; Prakapenka, V. B.; Talapin, D. V.; Schaller, R. D. Nano Lett. 2018, 18 (11), 6948-6953.

(25) Li, Q.; Lian, T. Chem. Sci. 2018, 9 (3), 728-734.

(26) Yakunin, S.; Protesescu, L.; Krieg, F.; Bodnarchuk, M. I.; Nedelcu, G.; Humer, M.; De Luca, G.; Fiebig, M.; Heiss, W.; Kovalenko, M. V. Nat. Commun. 2015, 6, 8056.

(27) Park, Y.-S.; Bae, W. K.; Baker, T.; Lim, J.; Klimov, V. I. Nano Lett. 2015, 15 (11), 7319-7328.

(28) Shaklee, K. L.; Leheny, R. F. Appl. Phys. Lett. 1971, 18 (11), 475.

(29) Shaklee, K. L.; Nahory, R. E.; Leheny, R. F. J. Lumin. 1973, 7 (C), 284-309.

(30) Samuel, I. D. W.; Turnbull, G. A. Chem. Rev. 2007, 107 (4), $1272-1295$.

(31) Dingle, R.; Shaklee, K. L.; Leheny, R. F.; Zetterstrom, R. B. Appl. Phys. Lett. 1971, 19 (1), 5-7.

(32) Valenta, J.; Pelant, I.; Linnros, J. Appl. Phys. Lett. 2002, 81 (8), 1396-1398.

(33) Cerdán, L.; Costela, A.; García-Moreno, I. J. Opt. Soc. Am. B 2010, 27 (9), 1874.

(34) Negro, L. D.; Bettotti, P.; Cazzanelli, M.; Pacifici, D.; Pavesi, L. Opt. Commun. 2004, 229 (1-6), 337-348.

(35) Cerdán, L.; Costela, A.; García-Moreno, I. J. Opt. Soc. Am. B 2010, 27 (9), 1874.

(36) Chow, W. W.; Wright, A. F.; Girndt, A.; Jahnke, F.; Koch, S. W. Appl. Phys. Lett. 1997, 71 (18), 2608.

(37) Delikanli, S.; Guzelturk, B.; Hernández-Martínez, P. L.; Erdem, T.; Kelestemur, Y.; Olutas, M.; Akgul, M. Z.; Demir, H. V. Adv. Funct. Mater. 2015, 25 (27), 4282-4289.

(38) Kelestemur, Y.; Dede, D.; Gungor, K.; Usanmaz, C. F.; Erdem, O.; Demir, H. V. Chem. Mater. 2017, 29 (11), 4857-4865.

(39) Bohnert, K.; Schmieder, G.; Klingshirn, C. Phys. Status Solidi B 1980, 98 (1), 175-188.

(40) Leuthold, J.; Mayer, M.; Eckner, J.; Guekos, G.; Melchior, H.; Zellweger, C. J. Appl. Phys. 2000, 87 (1), 618.

(41) Doussiere, P.; Garabedian, P.; Graver, C.; Bonnevie, D.; Fillion, T.; Derouin, E.; Monnot, M.; Provost, J. G.; Leclerc, D.; Klenk, M. IEEE Photonics Technol. Lett. 1994, 6 (2), 170-172.

(42) Arakawa, Y.; Yariv, A. IEEE J. Quantum Electron. 1986, 22 (9), $1887-1899$.

(43) Kirstaedter, N.; Schmidt, O. G.; Ledentsov, N. N.; Bimberg, D.; Ustinov, V. M.; Egorov, A. Y.; Zhukov, A. E.; Maximov, M. V.; Kop'ev, P. S.; Alferov, Z. I. Appl. Phys. Lett. 1996, 69 (9), 1226.

(44) Lam, S. Y.; Damzen, M. J. Appl. Phys. B: Lasers Opt. 2003, 77 (6-7), 577-584.

(45) Kim, H.; Schulte, N.; Zhou, G.; Müllen, K.; Laquai, F. Adv. Mater. 2011, 23 (7), 894-897.

(46) Lin, C. H.; Lafalce, E.; Jung, J.; Smith, M. J.; Malak, S. T.; Aryal, S.; Yoon, Y. J.; Zhai, Y.; Lin, Z.; Vardeny, Z. V.; Tsukruk, V. V. ACS Photonics 2016, 3 (4), 647-658.

(47) Kazes, M.; Lewis, D. Y. D. Y.; Ebenstein, Y.; Mokari, T.; Banin, U. Adv. Mater. 2002, 14 (4), 317-321.

(48) Geskus, D.; Aravazhi, S.; García-Blanco, S. M.; Pollnau, M. Adv. Mater. 2012, 24 (10), OP19-OP22.

(49) Sutherland, B. R.; Hoogland, S.; Adachi, M. M.; Kanjanaboos, P.; Wong, C. T. O.; McDowell, J. J.; Xu, J.; Voznyy, O.; Ning, Z.; Houtepen, A. J.; Sargent, E. H. Adv. Mater. 2015, 27 (1), 53-58.

(50) Hill, M. T.; Gather, M. C. Nat. Photonics 2014, 8 (12), 908918.

(51) Asada, M.; Miyamoto, Y.; Suematsu, Y. IEEE J. Quantum Electron. 1986, 22 (9), 1915-1921.
(52) Klimov, V. I.; Mikhailovsky, A. A.; Xu, S.; Malko, A.; Hollingsworth, J. A.; Leatherdale, C. A.; Eisler, H.; Bawendi, M. G. Science 2000, 290 (5490), 314-317.

(53) Yariv, A. Quantum Electronics, 3rd ed.; Wiley: New York, 1975.

(54) Pelton, M.; Ithurria, S.; Schaller, R. D.; Dolzhnikov, D. S.; Talapin, D. V. Nano Lett. 2012, 12 (12), 6158-6163.

(55) Lingk, C.; Plessen, G. von; Feldmann, J.; Stock, K.; Arzberger, M.; Böhm, G.; Amann, M.-C.; Abstreiter, G. Appl. Phys. Lett. 2000, 76 (24), 3507.

(56) Tomar, R.; Kulkarni, A.; Chen, K.; Singh, S.; Siebbeles, L.; Hodgkiss, J.; Geiregat, P.; Hens, Z. In Proceedings of the nanoGe Fall Meeting 2018; Fundació Scito: València, 2018.

(57) Klimov, V. I. Science 2000, 287 (5455), 1011-1013.

(58) Adachi, M. M.; Fan, F.; Sellan, D. P.; Hoogland, S.; Voznyy, O.; Houtepen, A. J.; Parrish, K. D.; Kanjanaboos, P.; Malen, J. A.; Sargent, E. H. Nat. Commun. 2015, 6 (1), 8694.

(59) Schmitt-Rink, S.; Chemla, D. S.; Miller, D. A. B. Phys. Rev. B: Condens. Matter Mater. Phys. 1985, 32 (10), 6601-6609.

(60) Amand, T.; Collet, J.; Razbirin, B. S. Phys. Rev. B: Condens. Matter Mater. Phys. 1986, 34 (4), 2718-2725. 